CARNETS DE Carnets de géographes

GÉOGRAPHES.

15 | 2021

Les dimensions spatiales du maintien de l'ordre

\title{
Mathieu Rigouste, La Domination Policière. Une violence industrielle
}

Paris : La Fabrique, 2012, 257 p.

\section{Alex Mahoudeau}

\section{OpenEdition}

\section{Journals}

Édition électronique

URL : https://journals.openedition.org/cdg/7523

DOI : $10.4000 /$ cdg.7523

ISSN : 2107-7266

\section{Éditeur}

UMR 245 - CESSMA

\section{Référence électronique}

Alex Mahoudeau, «Mathieu Rigouste, La Domination Policière. Une violence industrielle », Carnets de géographes [En ligne], 15 | 2021, mis en ligne le 15 avril 2021, consulté le 28 mai 2021. URL : http:// journals.openedition.org/cdg/7523; DOI : https://doi.org/10.4000/cdg.7523

Ce document a été généré automatiquement le 28 mai 2021.

\section{c) (ㅇ) $\ominus$}

La revue Carnets de géographes est mise à disposition selon les termes de la Licence Creative Commons Attribution - Pas d'Utilisation Commerciale - Pas de Modification 4.0 International. 


\title{
Mathieu Rigouste, La Domination Policière. Une violence industrielle
}

Paris : La Fabrique, 2012, 257 p.

\author{
Alex Mahoudeau
}

\section{RÉFÉRENCE}

Mathieu Rigouste, La Domination Policière. Une violence industrielle, Paris : La Fabrique, 2012, $257 \mathrm{p}$.

1 Paru en 2012, l'ouvrage de Mathieu Rigouste proposait d'analyser dans une perspective critique le fonctionnement et l'évolution de l'institution policière, ses pratiques, et leurs fondements matériels (p. 14). Neuf ans plus tard, le rôle de cette institution est au cœur de nombreuses controverses, particulièrement dans un contexte de montée des conflits en France et à l'étranger autour du rôle de cette institution. Cette intensification du conflit durant la seconde moitié des années 2010 est illustrée par l'émergence de mouvements construits autour de la dénonciation des violences policières (en France via des comités de familles de victimes, le comité Vérité et Justice pour Adama Traoré en tête, aux Etats-Unis via des mouvements comme Black Lives Matter, ou encore au Nigéria dans les mobilisations contre les "SARS », unités contre le vol, par exemple). Elle l'est aussi par la mise en avant de la répression policière dans des mobilisations touchant à d'autres sujets (particulièrement à Hong Kong vis-à-vis du mouvement pro-démocratie, mais également par exemple en France via le mouvement des «Gilets Jaunes »). Il a conduit durant l'année 2020 à l'ouverture d'un débat sur les opportunités et possibilités de réformer voire d'abolir l'institution policière. Ce débat s'est notamment structuré autour de l'enjeu de la violence, redonnant une actualité à cet ouvrage et à ses contributions sur le sujet. Compte-rendu du travail d'enquête mené par l'auteur sur ce sujet, l'ouvrage d'un peu plus de 250 pages se veut une proposition à la fois scientifique et militante, et parvient à remplir ces deux objectifs. 
2 Pourquoi revisiter ce texte (ou visiter, dans le cas de l'auteur de ces lignes), huit ans après sa parution et dans une revue de géographie ? Pourquoi le faire, à plus forte raison, quand l'auteur de l'ouvrage bénéficie d'une actualité éditoriale plus récente sur des thématiques similaires (Rigouste, 2020) ? A mon avis, c'est que l'ouvrage ne propose pas qu'une analyse de la police, mais qu'il analyse son action via un cadre théorique prenant au sérieux les dynamiques d'assignation de classe, de race, et de genre, que l'auteur parvient à mobiliser ici avec succès. Il contribue ainsi au développement des thématiques intéressant la géographie critique.

3 Le premier intérêt de l'ouvrage se trouve dans les réponses qu'il apporte à des interrogations contemporaines. À travers une enquête sociologique et historique, Mathieu Rigouste propose de mettre à jour les mécanismes à travers lesquels la police exerce sa fonction depuis la deuxième moitié $\mathrm{du} \mathrm{xx}^{\mathrm{e}}$ siècle, ainsi que la nature de cette fonction, à savoir le contrôle social et l'imposition de l'ordre. La thèse principale de l'ouvrage est que, loin d'être principalement un outil de lutte contre le crime, la police se développe sur cette période principalement comme une institution de contrôle social et politique, notamment via une réimportation de la logique coloniale en métropole (dynamique que l'auteur qualifie d'« endocoloniale »). Dans la conclusion de son ouvrage, l'auteur anticipe deux phénomènes à venir, à savoir l'extension des conflits sociaux mettant directement en cause les forces de police et le renforcement de la structuration de ce groupe social comme force politique. Ces deux phénomènes se sont en effet poursuivis. C'est également le cas des débats sur les tendances à la militarisation et à la privatisation qu'il évoque. La question des « violences policières " est régulièrement à l'agenda médiatique depuis la « révolte des banlieues " de 2005, mais généralement à travers le cadrage moralisateur des "bavures", et sans grand recul sur l'histoire ou le fonctionnement de l'institution.

4 L'enquête relève d'une sociologie critique, située, et compréhensive. Critique, car Mathieu Rigouste ne cache pas l'origine, l'orientation, et l'ambition de son ouvrage, se plaçant du côté des personnes victimes du rapport de domination et contre celui-ci. C'est en cela également que son travail est situé. Se positionnant dans «les strates supérieures des classes populaires»(p.9), il défend politiquement sa démarche au nom du témoignage de ses enquêtés, rencontrés à la suite de la parution de son ouvrage précédent - qui portait déjà sur les importations des catégories de pensée issues du colonialisme dans la construction d'un ordre sécuritaire en France au $\mathrm{xx}^{\mathrm{e}}$ siècle (Rigouste, 2009) - mais aussi d'une rigueur épistémique : "Malgré ce qu'en disent de nombreux sociologues de la police, la parole populaire n'est pas moins fiable que la parole policière : elle a au contraire l'avantage d'être réfutable et falsifiable» (p. 13). Compréhensive enfin, car l'auteur a mobilisé un vaste corpus d'archives issu d'activistes et d'intellectuels critiques; il a également analysé la parole des policiers eux-mêmes, à travers d'une part leurs publications (notamment celles de policiers s'exprimant sur des blogs), et d'autre part des entretiens qualitatifs menés avec des agents de police. L'auteur contribue ainsi à mettre à jour une institution violente au sens propre du terme :

Même les meurtres policiers sont pour une grande partie des applications d'idées et de pratiques portées par les différents niveaux de la hiérarchie policière et politique. Le mot « police » à lui seul contraint chaque fois qu'il est prononcé et par sa seule existence. Toute la police est violence jusque dans ses regards et ses silences. (p. 8) 
5 L'ouvrage est donc un compte-rendu d'enquêtes articulant des contributions théoriques (qui sont largement documentées par des renvois à la littérature) articulées à des éléments empiriques. L'auteur alterne ainsi entre des analyses d'évolutions au long cours de l'institution, ponctuées d'extraits d'entretiens, de billets de blogs, ou de documents d'archive, et des descriptions qui se veulent plus rapprochées et détaillées de scènes de violence, à travers des cas de «bavure » ou des «batailles » entre police et habitants, s'appuyant notamment sur le fond d'archives de Maurice Rajsfus, historien et journaliste ayant via son "Observatoire des libertés publiques » recensé à partir de 1993 les faits de violences policières en France. En retirant à la description de ces scènes le caractère souvent euphémisant qui peut occasionnellement leur être réservé, l'auteur parvient à illustrer la façon même dont s'impose la violence d'État. Ce rythme permet ainsi à Mathieu Rigouste de clarifier sa contribution théorique.

6 L'autre intérêt de ce texte est l'importance accordée à la dimension spatiale du travail de la police, à travers la territorialisation de son action, l'inscription scalaire de son travail, et surtout les réseaux dans lesquels elle s'insère. L'auteur analyse les processus de circulation des doctrines d'action de la police, de ses responsables, ses équipements, et ses modes d'action. L'auteur défend la thèse d'une continuité de la relation coloniale, particulièrement riche de ce point de vue: pour Mathieu Rigouste, la police, ses méthodes, et ses équipements, s'insèrent dans le cadre de ce qu'il appelle la " ségrégation endocoloniale ", c'est-à-dire la translation à l'intérieur des frontières de la métropole de techniques constituées dans le cadre de la domination coloniale. La police se développe ainsi par à-coups en touchant successivement les "damnés à l'intérieur» (en référence aux "damnés de la terre» de Frantz Fanon), donc les individus colonisés mais présents en métropole, et les «damnés de l'intérieur », leurs enfants et les catégories du prolétariat blanc qui leur sont associées. Dès son origine la police du quotidien sert en effet à séparer socialement et spatialement ces acteurs victimes de la répression du reste de la population. La domination policière se conçoit donc dans des espaces colonisés pour être ensuite importée au sein de la métropole, d'abord sur des populations criminalisées et marginalisées, puis progressivement au reste de la population. Exemple anecdotique mais parlant, le cas du préfet Pierre Bolotte, évoqué aux pages 35-36 : après avoir mené la répression sanglante d'une grève faisant suite à un meurtre raciste en Guadeloupe en 1968, il devient en 1969 préfet de Seine-Saint-Denis, avec pour mission de créer les premières brigades anti-criminalité (BAC). On voit dans l'ouvrage une succession d'histoires similaires. Dans son analyse du marché de la répression sur laquelle je reviens plus bas, l'auteur met à jour un autre aspect de cette circulation, non seulement internationale mais également entre forces de l'ordre : les techniques et équipements sont en effet transférés du secteur militaire, dans des zones de conflits situées dans les "Suds », vers le secteur policier dans les «Nords ", puis entre policiers à l'échelle internationale, comme par exemple dans le cas des lanceurs de balles non-perforantes, «passés » par la Palestine et l'Irlande du Nord, avant de "revenir » en France, par le biais d'unités antiterroristes, puis du maintien de l'ordre et enfin des brigades anticriminelles (pp. 109-111).

7 Cette lecture permet également un regard intéressant sur la façon dont l'État français après la Seconde Guerre Mondiale intègre la question de la race à ses politiques publiques, explicitement en la spatialisant via des pratiques de zonage, et implicitement comme catégorie d'action ordinaire des agents de police. Mathieu Rigouste revient ainsi sur la carrière de plusieurs agents qui passent successivement 
des Brigades Nord-Africaines (BNA) aux Brigades des Agressions et Violences (BAV), avant d'entrer dans le secteur de l'anti-criminalité dans des départements ciblés (notamment la Seine Saint-Denis), bénéficiant de promotions du fait de leur " connaissance des milieux nord-africains » (p. 31).

8 Il est fréquent d'évoquer la police comme acteur essentiel du rapport entre État et « quartiers » (catégorie spatiale employée comme euphémisme d'une catégorie raciale), et l'auteur prend la peine, notamment dans ses chapitres 2 «La ségrégation endocoloniale » et 3 «La tactique de la tension », de mettre au jour la façon dont les dispositifs de répression employés par la police reposent sur la séparation, le quadrillage, et l'envahissement de quartiers ainsi que sur la mise en place de mesures qui relèvent de la punition ou de l'intimidation collective. La présence de la police dans certains espaces s'apparente à une véritable occupation, marquée par le va-et-vient notamment des voitures et des hélicoptères toutes sirènes hurlantes sert à marquer dans l'espace et dans les corps la présence de l'institution et à rappeler implicitement à tous les habitants la nature du rapport entre eux et elle :

Des habitants du quartier de La Reynerie à Toulouse racontent, en 2012, comment depuis des années, sans interruptions, les équipes de BAC qui officient l'été dans la rue de Kiev allument chaque jour de la semaine leurs sirènes entre $4 \mathrm{~h}$ et $5 \mathrm{~h}$ du matin, sans aucune autre raison que de justifier leur présence. Elles font des allersretours dans cette rue qui fait caisse de résonance et réveillent ainsi chaque nuit plus de 2000 logements, et ce depuis près d'une dizaine d'années. (p. 152)

Comme le démontre l'auteur, cette relation aux « quartiers" n'est ni accidentelle ni extraite des rapports sociaux en général, elle vise la prise en charge d'un prolétariat en grande partie racisé : de la même façon que la pratique de l'îlotage se développe en parallèle de la politique de la ville, la domination policière accompagne sur le temps long les cycles de séparation, de désappropriation, et de dégagement de l'espace public qui touchent les classes populaires dans le cadre du processus de gentrification, mais également à travers des institutions qui sont aussi des lieux de mise à l'écart (centres de rétention administrative, prisons privatisées de façon croissante, etc.). Cette activité en parallèle permet la consolidation d'une forme de capital. La police, ainsi que le montre Mathieu Rigouste, n'est pas qu'affaire de répression, mais également de profits. Dans ses chapitres «Le marché de la coercition» et «Le renforcement du socioapartheid", il décrit l'intégration économique de cette institution à travers la construction d'un véritable complexe industrialo-policier ayant ses entreprisesfleuron. Ce complexe a ses produits emblématiques (à commencer par les lanceurs de balle de défense et les pistolets à impulsion électrique), ainsi que ses enjeux de concurrence internationale tant en matière d'équipement que d'expertise. L'action de la police et le déploiement de la violence sont nourris par ces innovations aussi bien qu'elles les nourrissent. Une police plus violente nécessite des équipements plus avancés, qui en retour permettent une répression plus accrue. Ce dernier point fait d'ailleurs écho aux propos d'agents de police et de responsables de BAC qui expliquent que la police n'a pas tant pour fonction de détecter que de créer les situations délictuelles. Cela passe par des provocations et l'entretien d'une tactique de la tension, qui justifie après coup le financement de leurs actions, dont le rôle demeure la séparation et la répression d'une partie des classes populaires, davantage que la réaction à des comportements « déviants ».

L'ensemble de ces éléments offre de la police le tableau d'une institution qui se constitue en «bricolant » au sein d'un référentiel général de défiance et de contrôle des 
classes dominées qui a peu à voir avec la vision idéalisée des " gardiens de la paix ». Ce référentiel s'ancre dans l'action de l'État indépendamment des alternances politiques, l'auteur critiquant l'idée selon laquelle un bord partisan serait « pro-police » tandis que l'autre ne le serait pas: plutôt qu'une droite "policière» et une gauche "antipolicière ", on voit plutôt une intensification de l'action policière au nom de l'efficacité sous la première, suivie d'une extension de ses effectifs au nom de la proximité sous la seconde. À travers une approche localisée des politiques publiques analysées au prisme des rapports de domination et des dynamiques sociales structurantes, Mathieu Rigouste produit ici un ouvrage qui partage nombre d'intérêts avec la géographie critique.

11 Le livre n'est pas sans limites. Plusieurs critiques peuvent venir à l'esprit à la lecture, par exemple la présentation très homogène de la police qui en ressort, et qui peut laisser sur sa faim la personne qui aborderait ce livre en espérant y trouver une dissection des différentes composantes de l'institution, ainsi que des statuts, des groupes, et des hiérarchies. D'autres ouvrages, comme le travail de Didier Fassin (2011) sur le fonctionnement au quotidien des brigades anti-criminalité, celui d'Alex Vitale (2017) sur la réforme policière, ou celui tout récent de Paul Rocher (2020) sur les armes non-létales, peuvent fournir des pistes intéressantes de continuation de la lecture sur ce sujet. Quoiqu'il en soit, alors que la police revient sur le devant de l'agenda médiatique, La Domination Policière n'a rien d'une lecture superflue.

\section{BIBLIOGRAPHIE}

FASSIN D. (2011), La force de l'ordre. Une anthropologie de la police des quartiers, Paris, Seuil.

RIGOUSTE M. (2009), L'ennemi intérieur. La généalogie coloniale de l'ordre sécuritaire dans la France contemporaine, Paris : La Découverte.

RIGOUSTE M. (2020), Un seul héros, le peuple. La contre-insurrection mise en échec par les soulèvements algériens de décembre 1960, Paris : Premiers Matins de Novembre.

ROCHER P. (2020), Gazer, mutiler, soumettre. Politique de l'arme non létale, Paris, La Fabrique.

VITALE A. (2017), The End of Policing, Londres/NewYork, Verso.

\section{AUTEURS}

\section{ALEX MAHOUDEAU}

Docteur en science politique, post-doctorant au groupe JEDI (Justice, Espace, Discriminations, Inégalités) du Labex « Futurs urbains » alex.mahoudeau[at]u-pem.fr 